\title{
From Dysarthria to Tetanus: Case Report and Diagnostic Considerations
}

\author{
Mariana Bilreiro, Luís Marote Correia \\ Serviço de Medicina Interna, Hospital Central do Funchal, Funchal, Portugal
}

Received: 15/12/2021

Accepted: 29/12/2021

Published: 20/01/2022

How to cite this article: Bilreiro M, Correia LM. From dysarthria to tetanus: case report and diagnostic considerations. EJCRIM 2022;9: doi:10.12890/2022_003131.

Conflicts of Interests: The authors declare there are no competing interests.

This article is licensed under a Commons Attribution Non-Commercial 4.0 License

\section{ABSTRACT}

Introduction: Tetanus is a vaccine-preventable disease caused by a neurotoxin produced by Clostridium tetani that proliferates in wound sites. Toxin interference with neuromuscular function leads to spasms. Trismus, risus sardonicus and opisthotonus are classic features, but tetanus can begin with subtler symptoms.

Case Description: An 80-year-old man presented with dysarthria. His medical history included hypertension and dyslipidaemia. No other neurological compromise was apparent on admission. Cranioencephalic computed tomography suggested pontine and mesencephalic ischaemia and stroke treatment was implemented. Two days later, the patient displayed dysphagia that required nasogastric intubation. The next day, he developed an apparent tonic seizure with respiratory distress refractory to diazepam and phenytoin, which required sedation and invasive mechanical ventilation. Ultimately, he manifested trismus and generalized spasms. Once the diagnosis of tetanus was established, he was given anti-tetanus immunoglobulin, tetanus toxoid vaccine and metronidazole. Magnetic resonance imaging did not reveal any brain injury. During his intensive care stay, he showed cardiovascular instability, developed nosocomial pneumonia, and required prolonged ventilator support and tracheostomy. He gradually improved during a 70-day hospital stay and regained his previous functional status.

Discussion: Dysarthria in an older patient with known cerebrovascular risk factors in addition to possible brainstem ischaemia contributed to an incorrect diagnosis of acute ischaemic stroke. Early manifestations of tetanus can mimic focal deficits. The limitations of brainstem computed tomography should be kept in mind.

Conclusion: Older patients present a broader range of signs suggesting tetanus, including a higher frequency of bulbar symptoms, on presentation. A careful anamnesis including previous vaccination history is key for identifying high-risk patients and to widen the differential diagnosis to include tetanus.

\section{LEARNING POINTS}

- Symptoms of tetanus include bulbar symptoms such as dysphagia and dysarthria in addition to muscle spasms.

- Older patients, especially if unvaccinated, are a vulnerable group in which a diagnosis of tetanus should be considered.

- One-slice non-contrast enhanced computed tomography of the brainstem is unreliable given the high frequency of technical artifacts.

\section{KEYWORDS}

Tetanus, dysarthria, delayed diagnosis, computed tomography

\section{INTRODUCTION}

Tetanus is caused by a neurotoxin produced by Clostridium tetani. Spores can enter the body through wounds or minor abrasions. Bacteria proliferation in the wound site leads to the production of toxin which binds to presynaptic membranes at neuromuscular junctions. 
The generalized type exhibits trismus and risus sardonicus as a result of facial muscle spasms which are typical early features and are often accompanied by dysphagia. Extensor spasms are typically stronger than flexor, causing characteristic opisthotonus. Severe cases exhibit altered consciousness, dysautonomia, cardiovascular instability and respiratory failure ${ }^{[1]}$.

Treatment includes wound cleaning and debridement, anti-toxin immunoglobulin, antibiotics such as metronidazole, control of muscle spasms with magnesium and baclofen, and supportive care. Untreated severe tetanus is often fatal. Preventive measures include wound care and tetanus toxoid vaccination. The latter is responsible for the decline in incidence in countries where it is efficiently implemented. Mortality in developed countries is less than $5 \%$. In 2015, there were an estimated 56,743 deaths due to tetanus worldwide ${ }^{[1]}$. As there are still no definitive diagnostic tests for tetanus, recognition based on clinical judgment is key for timely treatment.

\section{CASE DESCRIPTION}

An 80-year-old man presented to the emergency department unable to speak. His previous medical history included systemic arterial hypertension, hypercholesterolemia and benign prostatic hyperplasia. He had not received any vaccinations.

In the emergency department, his respiratory rate was 12 breaths per minute, blood pressure was 159/89 mmHg, and pulse was 70 beats per minute. He was conscious. Dysarthria was severe. The pupils were equal and reactive to light, and facial expression and somatic muscle strength were preserved. Muscle rigidity, clonus or spasms were not apparent. Gait was coordinated. Cranioencephalic computed tomography (CT) findings were interpreted as pontine and mesencephalic ischaemia (Fig. 1). He was admitted for presumed acute ischaemic stroke. Acetylsalicylic acid $250 \mathrm{mg}$ and atorvastatin $20 \mathrm{mg}$ by mouth were prescribed.

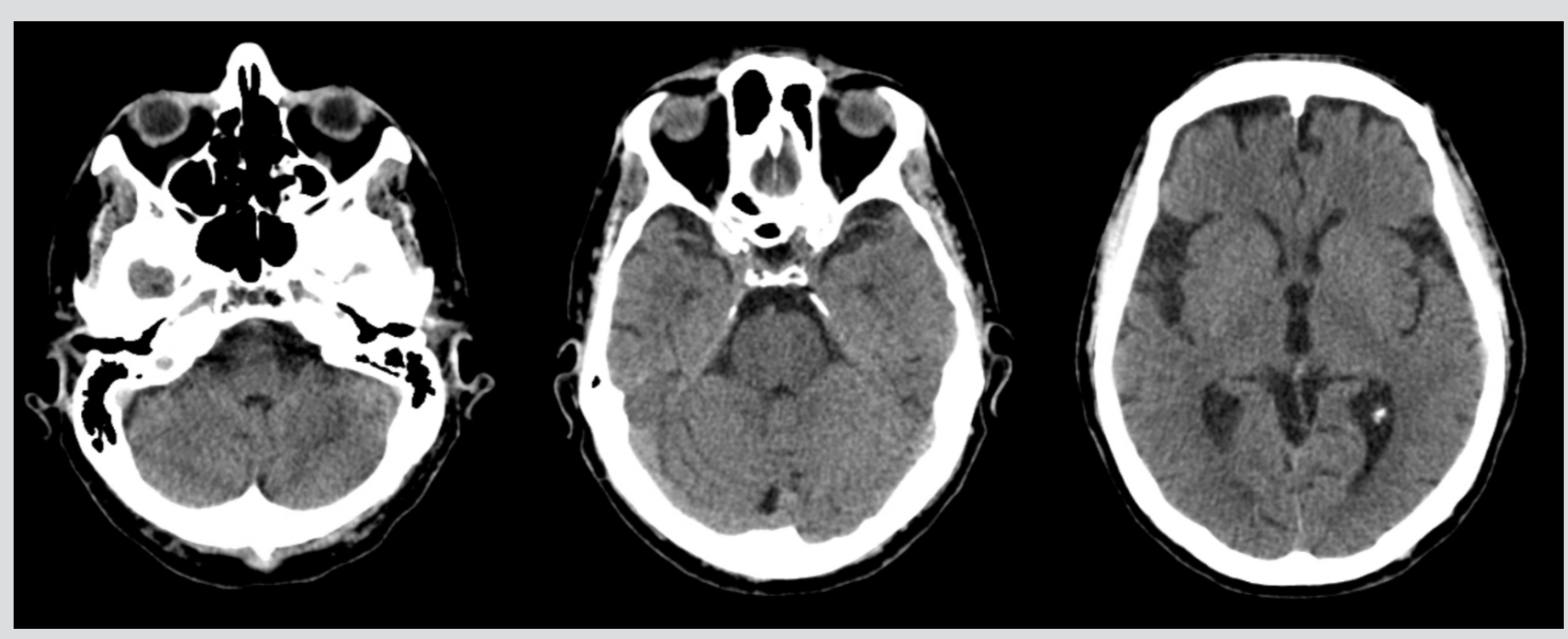

Figure 1. Non-contrast enhanced cranioencephalic computerized tomography showing hypodense artifacts in the pons and mesencephalon mimicking ischaemic lesions

On the third day, we identified dysphagia, so a nasogastric tube was placed to allow nutrition. The following day, the patient developed apparent tonic seizures and respiratory distress. An intra-hospital emergency team ineffectively repeated intravenous (IV) diazepam, and gave a phenytoin infusion. The patient was submitted to midazolam sedation, endotracheal intubation and invasive mechanical ventilation (IMV). Cranioencephalic CT re-evaluation showed the previous findings with no signs of infarction progression, cerebral oedema or haemorrhage. Blood analysis showed leucocytosis and rhabdomyolysis but results, including calcium levels, were otherwise normal (Table 1). Cerebrospinal fluid analysis was unremarkable.

On the fifth day, the patient had improved and was weaned off IMV. However, respiratory failure developed 12 hours after extubation. Trismus and platysma, abdominal and limb spasms were now evident. An entry wound at the base of the fourth interdigital space containing a $3 \mathrm{~mm}$ plant spike was cleaned. Neurology consult suggested a generalized tetanus diagnosis. Magnetic resonance imaging did not show any signs of brain injury (Fig. 2).

Human anti-tetanus immunoglobulin (6,000 U) was administrated intramuscularly. IMV was resumed, and continuous IV midazolam infusion was used for sedation and rocuronium for neuromuscular blockade. The patient received toxoid vaccine and a 14-day course of 


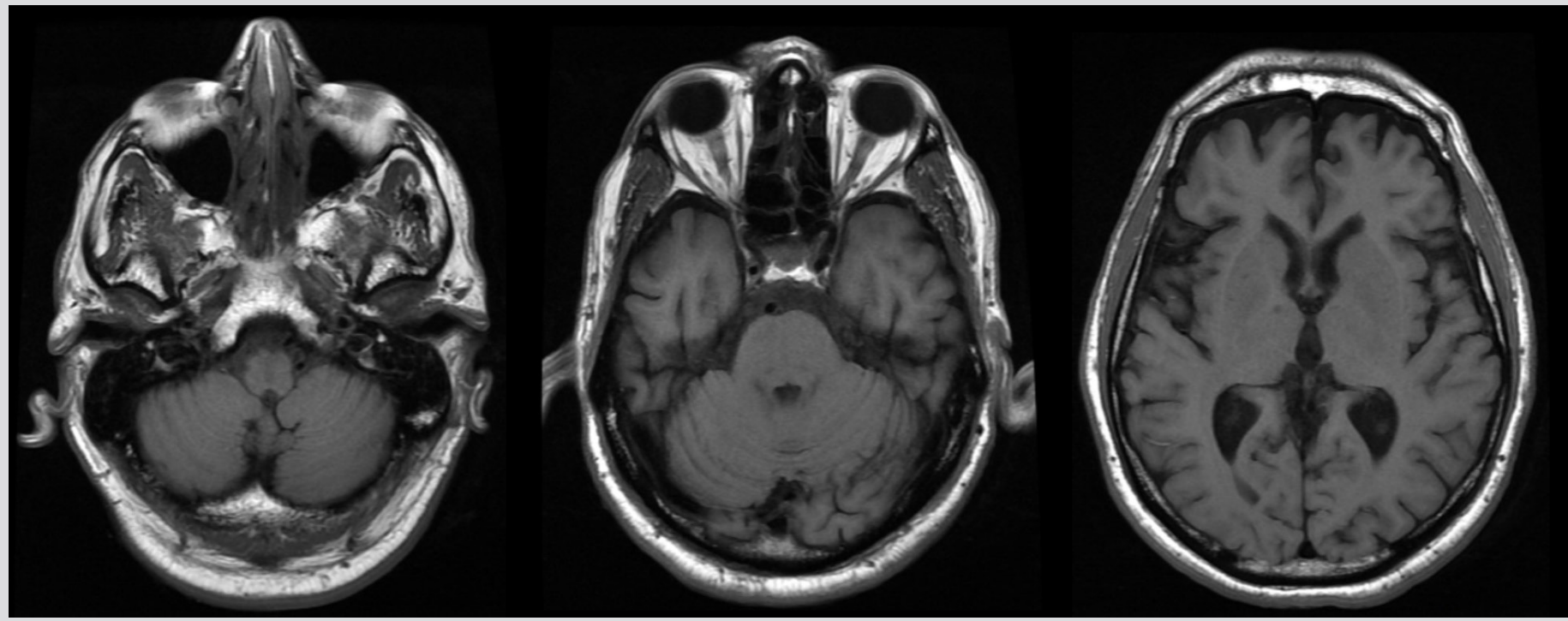

Figure 2. Cranioencephalic magnetic resonance imaging excluding lesions

\begin{tabular}{|c|c|c|}
\hline Parameter (unit) & Admission & Third day \\
\hline \multicolumn{3}{|l|}{ Peripheral blood } \\
\hline Leucocytes (per $\mu \mathrm{ll}$ ) & 9,500 & 13,500 \\
\hline Haemoglobin (g/dl) & 14.7 & 11.4 \\
\hline Platelets (per $\mu \mathrm{l})$ & 170,000 & 133,000 \\
\hline Glucose (mg/dl) & 106 & 149 \\
\hline Urea (mg/dl) & 50 & 50 \\
\hline Creatinine (mg/dl) & 1.29 & 1.13 \\
\hline Sodium (mEq/l) & 137 & 142 \\
\hline Potassium (mEq/l) & 4.3 & 4.5 \\
\hline Calcium (mEq/dl) & - & 9.0 \\
\hline Phosphorus (mg/dl) & - & 3.1 \\
\hline Magnesium (mg/dl) & - & 2.3 \\
\hline Creatine kinase (U/I) & 259 & 1,744 \\
\hline C-reactive protein (mg/l) & 1.9 & 4.8 \\
\hline \multicolumn{3}{|l|}{ Cerebrospinal fluid } \\
\hline Cells (per mm³) & - & 1 \\
\hline Glucose (mg/dl) & - & 67 \\
\hline Protein (mg/dl) & - & 17 \\
\hline Red blood cells (per mm $\mathrm{m}^{3}$ ) & - & $<1$ \\
\hline Chloride $(\mathrm{mmol} / \mathrm{l})$ & - & 117 \\
\hline
\end{tabular}

Table 1. Peripheral blood and cerebrospinal fluid analysis

metronidazole $500 \mathrm{mg}$ three times daily. Cardiovascular instability persisted for 48 hours and was characterized by several shifts in blood pressure (ranging from $81 / 43$ to $167 / 102 \mathrm{mmHg}$ ) and in heart rate (varying from 42 to 164 beats per minute). Spasms were controlled with magnesium sulphate up to a daily cumulative dose of $12,000 \mathrm{mg}$. Percutaneous tracheostomy was performed after 7 days of endotracheal intubation. Pneumonia caused by Pseudomonas aeruginosa was treated with a 10-day course of piperacillin+tazobactam 4,500 mg every 6 hours. 
The patient was transferred to a step-down unit on the 58th day of hospitalization and was able to speak again after the tracheostomy was closed. The nasogastric tube was removed once dysphagia was manageable with dietary adjustment. He was gradually weaned off non-invasive ventilator support as respiration improved. Muscle rigidity was managed with baclofen 10 mg three times a day, and intensive physical rehabilitation. After 70 days in hospital, the patient had regained his previous functional status. Vaccination resumed according to the national schedule. Figure 3 summarizes patient evolution.

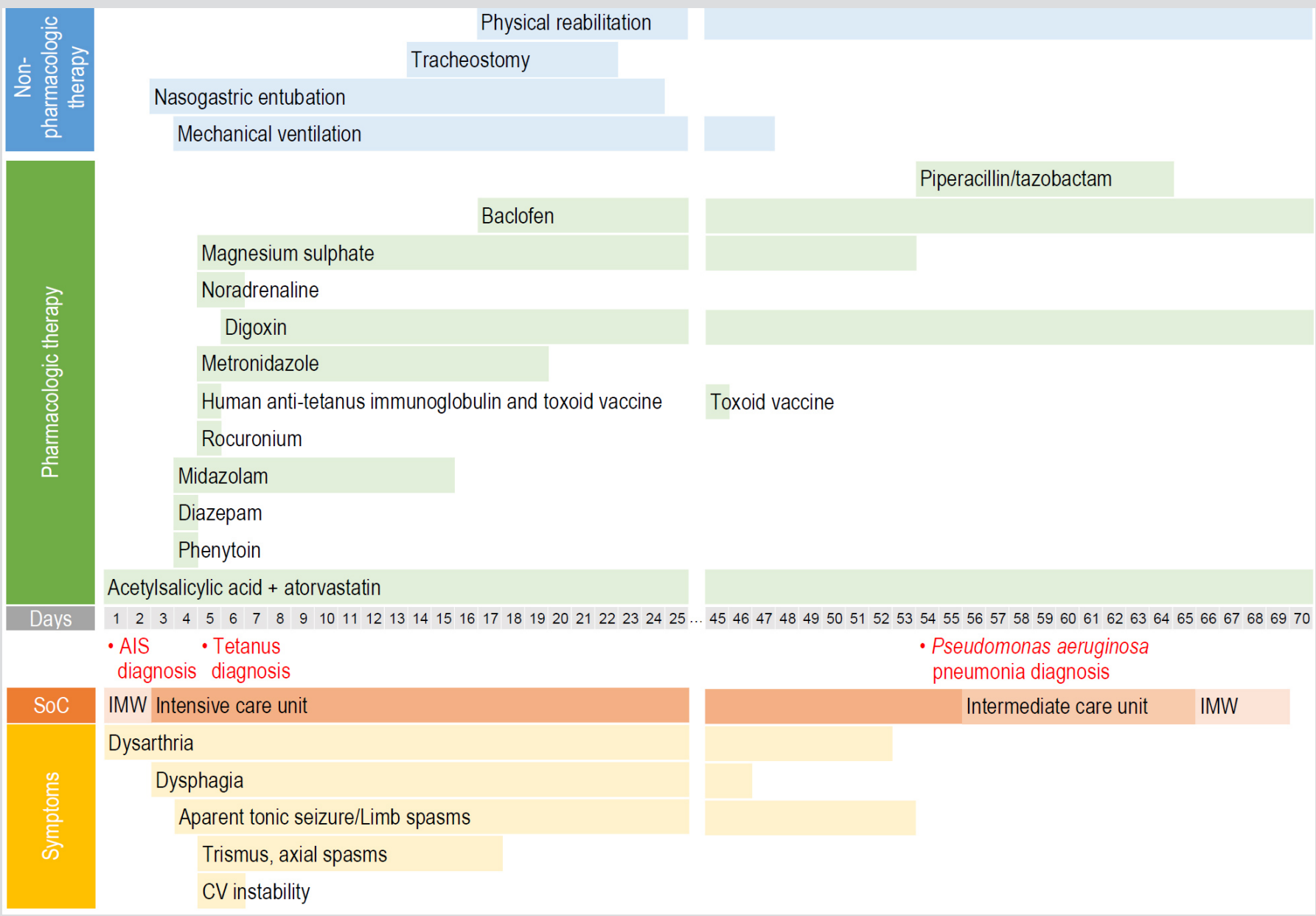

Figure 3. Treatment timeline

AIS, acute ischaemic stroke; CV, cardiovascular, IM, Internal Medicine; SoC, site of care

\section{DISCUSSION}

In our patient, a diagnosis of tetanus was delayed by two factors. First, the initial isolated dysarthria did not resemble the classic description of tetanus, and, when tetanus spasm occurred, it was concealed iatrogenically. Second, reliance on an incorrect radiological interpretation to explain dysarthria in a patient prone to cerebrovascular events and dismissal of anamnestic elements that could have broadened the differential diagnosis were due to confirmation bias.

Tetanus diagnosis is primarily semiological. Trismus was the most frequent clinical sign on admission in case series ${ }^{[2,3]}$. Alternative presentations and/or other symptoms vary across age groups. Patients aged 65 years or older were more likely to present with dysphagia/ dysarthria than younger individuals. In older people, bulbar paralysis manifestations were often more common than spasms at onset ${ }^{[3]}$. Local tetanus, mimicking focal deficits, can be an early feature of generalized tetanus. Pharyngeal and laryngeal discoordination may be a subtle initial manifestation ${ }^{[1]}$. As an isolated initial symptom, dysarthria is mentioned in one report that also led to a diagnosis of presumed cerebrovascular stroke ${ }^{[4]}$. 
The high incidence of cerebrovascular events among older patients, especially those with several risk factors, can suggest a different diagnosis. As described above, incorrect radiological information also delayed diagnosis. Evaluation of the brainstem and surrounding structures irrigated by posterior circulation through non-contrast enhanced CT is limited as the density of the bones of the skull base cause beam hardening and scatter artifacts ${ }^{[5]}$. Absence of other brainstem and cerebellar neurological deficits should warrant the use of a more accurate imaging technique. In our case, magnetic resonance imaging showed the CT findings were mere artifacts.

Vaccine-induced antibody titres decrease over time. In Europe, 25\% of those aged 65 years or older did not have protective antibody titres against tetanus ${ }^{[1]}$. Furthermore, adherence to anti-tetanus vaccination programs can be compromised by poor health education and a deficient healthcare system. Hence, older patients should be recognized as being more vulnerable to getting tetanus and high-risk individuals should be identified.

\section{CONCLUSION}

Older patients present a broader range of signs suggesting tetanus, including a higher frequency of bulbar symptoms. A careful anamnesis and vaccination history are key for identifying high-risk patients and to widen the differential diagnosis to include tetanus.

\section{REFERENCES}

Yen LM, Thwaites CL. Tetanus. Lancet 2019;393(10181):1657-1668.

Faust RA, Vickers OR, Cohn I Jr. Tetanus: 2,449 cases in 68 years at Charity Hospital. J Trauma 1976:16(9):704-712.

Weng WC, Huang WY, Peng TI, Chien YY, Chang KH, Ro LS, et al. Clinical characteristics of adult tetanus in a Taiwan medical center. J Formos Med Assoc 2011;110(11):705-710. Köhler A, Schaller P. Tetanus-eine Differentialdiagnose des zerebrovaskulären Insultes [Tetanus-differential diagnosis of cerebrovascular stroke]. Praxis 2001;90(15):643646.

5. Caldwell J, Heran MKS, McGuinness B, Barber PA. Imaging in acute ischaemic stroke: pearls and pitfalls. Pract Neurol 2017;17(5):349-358. 